\title{
Resolution of the Identity of 'Candidatus Liberibacter' Species From Huanglongbing-Affected Citrus in East Africa
}

Ronel Roberts, Plant Microbiology Division, Agricultural Research Council-Plant Protection Research, Pretoria 0001, South Africa; Glynnis Cook and Tim G. Grout, Citrus Research International, Nelspruit 1200, South Africa; Fathiya Khamis, Ivan Rwomushana, and Peterson W. Nderitu, International Centre of Insect Physiology and Ecology, Nairobi, Kenya; Zuberi Seguni and Christopher L. Materu, Mikocheni Agricultural Research Institute, Dar es Salaam, Tanzania; Chanel Steyn, Citrus Research International, Nelspruit 1200, South Africa; Gerhard Pietersen, Plant Microbiology Division, Agricultural Research Council-Plant Protection Research, Pretoria 0001, South Africa; Sunday Ekesi, International Centre of Insect Physiology and Ecology, Nairobi, Kenya; and Hendrick F. le Roux ${ }^{\dagger}$, Citrus Research International, Nelspruit 1200 , South Africa

\begin{abstract}
'Candidatus Liberibacter asiaticus', the bacterium associated with citrus Huanglongbing (HLB), was reported from Uganda and tentatively from Tanzania, posing a threat to citriculture in Africa. Two surveys of citrus expressing typical HLB symptoms were conducted in Uganda, Kenya, and Tanzania to verify reports of ' $\mathrm{Ca}$. L. asiaticus' and to assess the overall threat of HLB to eastern and southern African citrus production. Samples were analyzed for the presence of 'Candidatus Liberibacter' species by real-time PCR and partial sequencing of three housekeeping genes, 16S rDNA, $r p l J$, and omp. ' $C a$. L. africanus', the bacterium historically associated with HLB symptoms in Africa, was detected in several samples. However, samples positive in real-time PCR for ' $\mathrm{Ca}$. L. asiaticus'

were shown not to contain ' $C a$. L. asiaticus' by sequencing. Sequences obtained from these samples were analogous to ' $\mathrm{Ca}$. L. africanus subsp. clausenae', identified from an indigenous Rutaceae species in South Africa, and not to ' $\mathrm{Ca}$. L. asiaticus'. Results indicate a nontarget amplification of the real-time assay and suggest that previous reports of ' $\mathrm{Ca}$. $\mathrm{L}$. asiaticus' from Uganda and Tanzania may be mis-identifications of ' $\mathrm{Ca}$. L. africanus subsp. clausenae'. This subspecies was additionally detected in individual Diaphorina citri and Trioza erytreae specimens recovered from collection sites. This is the first report of ' $\mathrm{Ca}$. L. africanus subsp. clausenae' infecting citrus and being associated with HLB symptoms in this host.
\end{abstract}

Three phloem limited bacterial species, 'Candidatus Liberibacter asiaticus', ' $\mathrm{Ca}$. L. africanus', and ' $\mathrm{Ca}$. L. americanus' are associated with the devastating disease of citrus, Huanglongbing (HLB) (Jagoueix et al. 1994; Teixeira et al. 2005). Symptoms characteristic of HLB include blotchy mottling of mature leaves, reduction in fruit yield and quality, and overall tree decline (Kapur et al. 1978). ' $C a$. L. asiaticus' is the most widely distributed, being reported from citrus crops in Asia (Garnier and Bové 1996), the Mascarene islands (Garnier et al. 1996), the Americas (Coletta-Filho et al. 2004; Halbert 2005), and Papua New Guinea (Weinert et al. 2004). HLB symptoms first reported from citrus in Brazil were found to be associated with ' $\mathrm{Ca}$. L. americanus' (Teixeira et al. 2005).

The African continent remained free from ' $\mathrm{Ca}$. L. asiaticus' until 2010, when detection of this bacterium was reported from citrus trees in northern Ethiopia (Saponari et al. 2010). Despite the recent introduction of ' $\mathrm{Ca}$. L. asiaticus' onto the African continent, ' $\mathrm{Ca}$. L. africanus' is historically known to be the most prevalent Liberibacter species on citrus in Africa and was first observed in South African citrus orchards in the late 1920s (Oberholzer et al. 1963). The disease associated with ' $\mathrm{Ca}$. L. africanus' in South Africa is locally known as African greening (Oberholzer et al. 1963). Since its initial discovery, ' $C a$. L. africanus' has been associated with citrus in 15 other African countries and the Mascarene islands (Bové 2014; da Graça 1991; Garnier and Bové 1996; Garnier et al. 1996). In addition to ' $\mathrm{Ca}$. L. africanus', five subspecies of ' $\mathrm{Ca}$. L. africanus' have been

Corresponding author: Glynnis Cook; glynnis@cri.co.za

${ }^{\dagger}$ Deceased 4 October 2016

*The $\boldsymbol{e}$-Xtra logo stands for "electronic extra" and indicates that one supplementary table is published online.

Accepted for publication 6 April 2017.

C 2017 The American Phytopathological Society identified from indigenous rutaceous trees in South Africa, i.e., ' $C a$. L. africanus subsp. capensis' (Garnier et al. 2000), ' $C a$. L. africanus subsp. clausenae', ' $C a$. L. africanus subsp. vepridis', 'Ca. L. africanus subsp. zanthoxyli' (Roberts et al. 2015), and ' $C a$. L. africanus subsp. tecleae' (Roberts and Pietersen 2016). Currently, little is known about these ' $C a$. L. africanus' subspecies regarding their host range, transmission, and disease causing potential to citrus.

The impact of HLB is devastating as is demonstrated by the destruction of over 100 million commercially grown citrus trees in Asia (Zhang et al. 2010). This crippling effect was similarly experienced in São Paulo State of Brazil with the loss of a million citrus trees within 3 years (Gottwald et al. 2007). The more recent introduction of ' $\mathrm{Ca}$. L. asiaticus' in Florida, U.S.A., was accompanied by significant production decline within the first decade since the initial discovery of HLB in 2005 (Halbert 2005) and this was followed by the introduction of HLB into Texas (da Graça et al. 2015).

Despite causing severe losses up until the late 1970s (Buitendag and von Broembsen 1993; Schwarz 1967), the incidence of HLB in South Africa is maintained at economically acceptable levels through integrated management strategies involving inoculum removal and vector control (Pretorius and van Vuuren 2006). The effect of ' $\mathrm{Ca}$. L. africanus' has been less devastating than disease occurrences associated with ' $\mathrm{Ca}$. L. asiaticus'. This can be attributed to the greater temperature sensitivity of ' $\mathrm{Ca}$. L. africanus' (Schwarz and Green 1972) as well as that of its natural vector, Trioza erytreae del Guercio (Hemiptera: Triozidae) (Catling 1969; McClean and Oberholzer 1965), compared with ' $\mathrm{Ca}$. L. asiaticus' and its natural liviid vector Diaphorina citri Kuwayama (Hemiptera: Liviidae) (Capoor et el. 1967) limiting the occurrence of HLB to cooler production areas. These factors combined with the effective control of T. erytreae makes it a manageable disease in Africa.

In 2012, 'Ca. L. asiaticus' was reportedly detected in Uganda (Kalyebi et al. 2015) and was subsequently followed by a report of the presence of $D$. citri in Tanzania, with 'C $a$. L. asiaticus' suggested as the causal agent of HLB at some locations surveyed (Shimwela et al. 2016). The detection of ' $\mathrm{Ca}$. L. asiaticus' on citrus outside of Asia was preceded by the discovery of D. citri (Capoor et al. 
1967) in all incidences (Davis et al. 2000; Gravena et al. 1996; Knapp et al. 1998), except for the discovery of ' $\mathrm{Ca}$. L. asiaticus' in Ethiopia where $D$. citri is not reported yet (Saponari et al. 2010). The presence of $\mathrm{D}$. citri in Tanzania therefore suggests that ' $\mathrm{Ca}$. L. asiaticus' can potentially be spread on the African mainland by this vector if the two agents meet, thereby affecting warmer production areas previously unaffected by HLB. Additionally, ' $\mathrm{Ca}$. L. asiaticus' could potentially also be spread by $T$. erytreae as this vector can transmit both ' $C a$. L. africanus' and ' $C a$. L. asiaticus' (Lallemand et al. 1986; Massonie et al. 1976). Therefore, the occurrence of both ' $\mathrm{Ca}$. L. asiaticus' and its naturally associated vector from Asia poses a significant threat to African citrus production and needs to be clarified in order to initiate mitigation strategies.

This study was initiated to clarify previous reports of ' $\mathrm{Ca}$. L. asiaticus' from sites in Tanzania and Uganda where the bacterium had previously been reported and to establish how widely the pathogen had spread. The study also sought to establish the identity of other Liberibacters responsible for causing HLB symptoms in Kenya, Tanzania, and Uganda and their ecological limits.

\section{Materials and Methods}

Surveys. A field survey for HLB and associated vectors $D$. citri and T. erytreae was conducted in March 2016 in Tororo and Budaka districts of Uganda, where ' $\mathrm{Ca}$. L. asiaticus', associated with $T$. erytreae, had reportedly been detected (Kalyebi et al. 2015). Additionally, the adjacent counties of Kisumu, Kericho, and Nakuru in western Kenya were also surveyed to detect any possible occurrence of HLB in the Rift Valley.

A second survey was conducted in May 2016 from north of Malindi district on the eastern coast of Kenya, extending along coastal highways through the counties of Malindi, Kilifi, Mombasa, and Diani, turning inland to Kwale County. It continued further south through Msambweni and Lunga Lunga to the Tanzanian border, then through Tanga, Muheza, and Handeni and branching southwest to Morogoro where the unconfirmed report of ' $\mathrm{Ca}$. L. asiaticus' by Shimwela et al. (2016) originated. The survey also extended south of Dar es Salaam to Kibiti and east to Zanzibar.

These surveys included sampling citrus trees grown for commercial or subsistence use and showing blotchy mottle on the leaves, typical of HLB symptoms. GPS coordinates of sample sites were recorded (Supplementary Table S1). Two separate sets of leaf samples were collected at each site and samples placed in sealed plastic bags. All citrus trees were additionally scouted for the presence of either D. citri or T. erytreae.

Separate analyses of the leaf samples were conducted by three laboratories for Liberibacter identification for the first survey. One sample set was taken to the International Centre of Insect Physiology and Ecology (icipe) and the other shipped to South Africa under permit for the importation of controlled goods as issued by the South African Directorate of Plant Health. Analysis of this sample set was conducted by two laboratories in South Africa, namely the Agricultural
Research Council-Plant Protection Research (ARC-PPR) and Citrus Research International (CRI). Samples from the second survey were analyzed by the two laboratories in South Africa.

Collection and identification of Trioza erytreae and Diaphorina citri samples. T. erytreae adults were collected in Kericho district in Kenya and D. citri from the Morogoro region in Tanzania on citrus plants displaying mottling. Adults were morphologically identified using forewing venation and patterns.

DNA isolation. Genomic DNA was extracted from citrus petioles and midribs using either a CTAB method as described by Doyle and Doyle (1990) or the Isolate II Plant DNA extraction kit (Bioline, U.K.) following the manufacturer's protocol. DNA was extracted from $T$. erytreae and $D$. citri adults using a modified CTAB method. Single adults were macerated using a pipette tip in $250 \mu \mathrm{l} \mathrm{CTAB}$ buffer in a $1.5 \mathrm{ml}$ reaction tube and the macerate was incubated at $60^{\circ} \mathrm{C}$ for $30 \mathrm{~min}$. An equal volume of chloroform was added, the suspension vortexed and centrifuged at 13,000 rpm for $5 \mathrm{~min}$. Nucleic acids were precipitated with ethanol, pelleted by centrifugation, washed in $75 \%$ ethanol, and the final pellet resuspended in $15 \mu \mathrm{l}$ distilled water.

Real-time PCR. Real-time PCR for the specific detection of both ' $C a$. L. africanus' and ' $\mathrm{Ca}$. L. asiaticus' was done using primers and probe described by $\mathrm{Li}$ et al. (2006). Reactions were set up by the addition of $250 \mathrm{ng}$ of DNA to a final reaction volume of $20 \mu \mathrm{l}$ containing $2 \times$ KAPA Probe Fast Master Mix Universal (KAPA Biosystems), $400 \mathrm{nM}$ per primer and $250 \mathrm{nM}$ probe. Real-time PCR reactions were run on a Rotor-Gene Q (Qiagen) with the following parameters: initial denaturation of $5 \mathrm{~min}$ at $95^{\circ} \mathrm{C}$ followed by 40 cycles at $95^{\circ} \mathrm{C}$ for $5 \mathrm{~s}$ and $62^{\circ} \mathrm{C}$ for $20 \mathrm{~s}$. Crossing threshold values based on fluorescent signals were determined using Rotor-gene Q version 2.3.1.49 software. A threshold of $\mathrm{Ct}<35$ was used as cutoff value for positive Liberibacter detection.

Endpoint PCR and Direct Sequencing. The identity of the Liberibacter species present per sample were verified by direct sequencing of endpoint PCR amplicons. These included amplicons of partial regions of the 16S rDNA using primers OA1 and OI2c (Jagoueix et al. 1996), the ribosomal protein $\mathrm{J}(\mathrm{rplJ})$ using primers A2 and J5 (Hocquellet et al. 1999), and the outer membrane protein (omp) gene region using primers HP1inv and OMP8inv (Bastianel et al. 2005). Primer details are provided in Table 1. Reactions were done using either DreamTaq Green PCR Master Mix (Thermo Scientific), GoTaq G2 Hot Start Green Master Mix (Promega Corp.), or My Taq DNA Polymerase (Bioline).

PCR amplification products were purified enzymatically using exonuclease I (Thermo Scientific) and Fast AP (Thermo Scientific) or alternatively using either the Zymoclean Gel DNA recovery kit (Zymo Research) or the Isolate II PCR and Gel Kit (Bioline, U.K.) according to manufacturers' instructions. Purified products were bidirectional sequenced. Low quality bases were removed and the overlapping sequences were aligned using BioEdit version 7.2.5 (Hall 1999).

Phylogenetic analysis. Sequence datasets for each gene region were analyzed along with relevant reference sequences from related

Table 1. Primer sequences, expected product size, and annealing temperatures used for Liberibacter spp. determination and 'Candidatus Liberibacter africanus' and ' $\mathrm{Ca}$. L. africanus subsp. clausenae' specific detection

\begin{tabular}{|c|c|c|c|c|c|}
\hline Primer & Sequence $5^{\prime}$ - $3^{\prime}$ & Target gene & Source & PCR product size (bp) & Annealing temp $\left({ }^{\circ} \mathrm{C}\right)$ \\
\hline A2 & TATAAAGGTTGACCTTTCGAGTTT & $\mathrm{rplA} / \mathrm{rplJ}$ & Hocquellet et al. (1999) & $703^{\mathrm{a}}$ & 58 \\
\hline J5 & ACAAAAGCAGAAATAGCACGAACAA & & & $669^{\mathrm{b}}$ & \\
\hline OA1 & GCGCGTATTTTATACGAGCGGCA & $16 \mathrm{~S}$ & Jagoueix et al. (1996) & 1,160 & 62 \\
\hline $\mathrm{OI} 2 \mathrm{c}$ & GCCTCGCGACTTCGCAACCCAT & & & & \\
\hline HP1inv & GGGTCACGGGTTTTATGAATTTGTTG & Omp & Bastianel et al. (2005) & 1,400 & 55 \\
\hline OMP8inv & CTAAAATCAAGCTCACGACGAATCAC & & & & \\
\hline Laf F & TCTCCGACGCGTATCAATCT & Omp & This study & 250 & 65 \\
\hline Laf $\mathrm{R}$ & CGCGATGACACCTTAACTGC & & & & \\
\hline $\mathrm{LafCl}^{\mathrm{c}} \mathrm{F}$ & CGGTAGTCCTCACTCTTTCGTA & Omp & This study & 199 & 65 \\
\hline LafCl R & ATGAATCACCGAAACAGCGG & & & & \\
\hline
\end{tabular}


citrus-associated Liberibacter species obtained from GenBank. Alignments were done using MAFFT (Katoh et al. 2002), which were subsequently trimmed using BioEdit. Maximum-likelihood phylogeny analyses were done with MEGA software 7.0.14 (Kumar et al. 2016) by inferring best-fit substitution models.

Specific endpoint PCR assays for detection of ' $\mathrm{Ca}$. $\mathrm{L}$. africanus' and ' $\boldsymbol{C a}$. L. africanus subsp. clausenae'. Primers were designed for endpoint PCR for the specific detection of both ' $\mathrm{Ca}$. L. africanus' and ' $\mathrm{Ca}$. L. africanus subsp. clausenae' based on available sequences in the omp gene region. Primer sequences, expected amplicon sizes, and their relative annealing temperatures are presented in Table 1. Reaction mix and cycling parameters were as described by Roberts et al. (2015).

Specificity of ' $\mathrm{Ca}$. L. asiaticus' and ' $\mathrm{Ca}$. L. africanus' real-time PCR detection assay. The specificity of the $\mathrm{Li}$ et al. (2006) primers and probe was determined by testing the various ' $\mathrm{Ca}$. L. africanus' subspecies in both the ' $\mathrm{Ca}$. L. africanus' and the ' $\mathrm{Ca}$. L. asiaticus' assay. The real-time PCR assay reaction components and cycling conditions were as described above for real-time detection. Samples containing ' $\mathrm{Ca}$. L. africanus' in Citrus sinensis and various ' $\mathrm{Ca}$. L. africanus' subspecies in rutaceous hosts, obtained from various sites around South Africa, were used. The ' $\mathrm{Ca}$. L. asiaticus' positive sample was collected in Ethiopia in 2014 under permit. Three samples per Liberibacter subspecies were included and reactions were performed in triplicate.

\section{Results}

Survey. In total, 61 sites were inspected, but leaf samples were only taken from citrus when leaves expressed symptoms suspected to be HLB, except for some low altitude sites in Tanzania. The citrus trees sampled were either sweet orange (C. sinensis [L.] Osbeck) or rough lemon (C. jambhiri Lush.), which was the only rootstock in use. Often, watershoots from the rootstock were intertwined in the canopy so foliage comprised a mixture of both species. Most trees had been harvested at the time of the surveys and if there was no new growth present, it was not always obvious what the trees were. No citrus samples showing blotchy-mottle leaf symptoms were observed in the lower lying coastal regions and mottling was seldom seen on trees in full sun at higher altitudes, but usually on trees that were shaded by other larger trees. Forty-one citrus leaf samples were collected during the two surveys. Of these samples, 18 originated from Uganda and six from western Kenya in the first survey. These sites were at elevations at or above $1,090 \mathrm{~m}$. The remaining 17 samples were collected from low to high altitude regions (19 to $668 \mathrm{~m}$ ) in Tanzania during the second survey. The sampling sites are listed in Table 2.

'Ca. L. africanus' and 'Ca. L. asiaticus' real-time PCR. Of the 41 citrus samples, 10 were positive for ' $C a$. L. africanus' only, 16 for ' $C a$. L. asiaticus' only, and three samples from Tanzania tested positive in both ' $\mathrm{Ca}$. L. africanus' and ' $\mathrm{Ca}$. L. asiaticus' specific real-time PCR assays (Table 2), suggesting that these samples were coinfected with at least two Liberibacters. In total, 29 samples tested positive for a Liberibacter sp.

Phylogenetic analysis. Phylogenetic analysis of $16 \mathrm{~S}$ rDNA sequences obtained for the Liberibacter positive samples showed that 14 samples clustered with known ' $\mathrm{Ca}$. L. africanus' sequences sharing $100 \%$ nucleotide identities with other ' $\mathrm{Ca}$. L. africanus' $16 \mathrm{~S}$ rDNA sequences. Sixteen samples clustered with 16S rDNA sequences of ' $C a$. L. africanus subsp. capensis', ' $C a$. L. africanus subsp. clausenae', and ' $\mathrm{Ca}$. L. africanus subsp. vepridis' (Fig. 1) sharing $100 \%$ sequence similarity to these three ' $\mathrm{Ca}$. L. africanus' subspecies and sequence similarities of 99.2, 99.3, 99.5, 98.2, and $94.7 \%$ to ' $\mathrm{Ca}$. L. africanus', ' $\mathrm{Ca}$. L. africanus subsp. zanthoxyli', 'Ca. L. africanus subsp. tecleae', ' $\mathrm{Ca}$. L. asiaticus', and ' $\mathrm{Ca}$. L. americanus', respectively, across $1,076 \mathrm{bp}$. None of the samples clustered with ' $C a$. L. asiaticus' $16 \mathrm{~S}$ rDNA sequences.

However, based on phylogenetic analysis of the $r p l J$ sequences, 13 samples clustered with ' $\mathrm{Ca}$. L. africanus', sharing $100 \%$ nucleotide identities with known ' $C a$. L. africanus' sequences, and 17 samples clustered nearest to ' $\mathrm{Ca}$. L. africanus subsp. clausenae' sharing
98.2\% nucleotide identity with ' $C a$. L. africanus subsp. clausenae' sequences obtained from Clausena anisata Hook. f. ex Benth (Fig. 2). Eleven nonsynonymous single nucleotide polymorphisms (SNPs) were observed between ' $\mathrm{Ca}$. L. africanus subsp. clausenae' and the ' $C a$. L. africanus subsp. clausenae' from citrus across $681 \mathrm{bp}$. These SNPs occurred at the same nucleotide positions among all citrus samples testing positive for this subspecies.

Phylogeny based on omp sequences supported the grouping of $r p l J$ sequences with 13 samples containing sequences identical to ' $C a$. L. africanus' and 16 samples showing sequence homology to 'Ca. L. africanus subsp. clausenae' (Fig. 3). A single sample consistently gave a mixed sequence for this gene region and could not be assessed phylogenetically. Sequences obtained for ' $\mathrm{Ca}$. L. africanus subsp. clausenae' from citrus shared $99 \%$ sequence identity with 'Ca. L. africanus subsp. clausenae' omp sequences from C. anisata. Fifteen SNPs were observed across the 1,143 bp omp sequence.

Representative sequences for the ' $\mathrm{Ca}$. L. africanus subsp. clausenae' 16S rDNA, rplJ, and omp gene regions were submitted to GenBank under the accession numbers KX839155, KX770998, and KX904936, respectively.

Analysis of a separate set of samples analyzed at icipe supported the above findings. In Uganda, both ' $\mathrm{Ca}$. L. africanus' and ' $\mathrm{C} a$. L. africanus subsp. clausenae' were detected in the Budaka district and ' $\mathrm{Ca}$. L. africanus subsp. clausenae' in the Tororo district. Similarly, only ' $\mathrm{Ca}$. L. africanus subsp. clausenae' was detected in samples collected at Soroget, Kisumu, and Kericho counties in Kenya. No ' $\mathrm{Ca}$. L. asiaticus' was detected.

Specificity of ' $\mathrm{Ca}$. L. asiaticus' and ' $\mathrm{Ca}$. L. africanus' real-time PCR detection assay. Survey samples containing sequences homologous to ' $\mathrm{C} a$. L. africanus subsp. clausenae', and not ' $\mathrm{Ca}$. L. asiaticus', tested positive for ' $\mathrm{Ca}$. L. asiaticus' following the ' $\mathrm{Ca}$. L. asiaticus' real-time PCR assay of Li et al. (2006) (Table 2), suggesting that this assay also amplifies from ' $\mathrm{Ca}$. L. africanus subsp. clausenae' and so the use of the ' $\mathrm{Ca}$. L. asiaticus' primers can lead to misidentification of Liberibacter(s) present in a sample. To verify this nontarget amplification, ' $\mathrm{Ca}$. L. africanus' and ' $\mathrm{Ca}$. L. asiaticus' real-time PCR assays were performed using samples that were previously characterized as containing one of the ' $\mathrm{Ca}$. L. africanus' subspecies. Samples of rutaceous hosts containing various ' $\mathrm{Ca}$. L. africanus' subspecies tested positive with both ' $\mathrm{Ca}$. L. africanus' and ' $\mathrm{Ca}$. L. asiaticus' specific real-time PCR assays, with the exception of a single 'Ca. L. africanus subsp. zanthoxyli' sample (Table 3). The Ct values obtained for the various subspecies of ' $\mathrm{Ca}$. L. africanus' were consistently lower in the ' $\mathrm{Ca}$. L. asiaticus' assay compared with the ' $\mathrm{Ca}$. L. africanus' assay for all the rutaceous samples tested.

Specific detection of ' $\mathrm{Ca}$. L. africanus' and ' $\mathrm{Ca}$. L. africanus subsp. clausenae'. To determine whether a single tree can be coinfected with ' $\mathrm{Ca}$. L. africanus' and ' $\mathrm{Ca}$. L. africanus subsp. clausenae', primers were designed for the specific detection of both ' $\mathrm{Ca}$. L. africanus' and ' $\mathrm{Ca}$. L. africanus subsp. clausenae' based on $o m p$ sequences (Table 1). The primer sets described herein were shown to react only with their intended target and no cross reaction was observed for any of the other known citrus infecting Liberibacter species or subspecies (Fig. 4), indicating that the Liberibacter omp gene region is a suitable target for differentiating between the various Liberibacter species and subspecies known.

Using these primers, 10 survey samples tested positive for ' $\mathrm{Ca}$. $\mathrm{L}$. africanus' only, 15 samples were positive for ' $\mathrm{Ca}$. L. africanus subsp. clausenae' only, and five samples gave amplification products for both ' $\mathrm{Ca}$. L. africanus' and ' $\mathrm{Ca}$. L. africanus subsp. clausenae' (Table 2). 'Ca. L. africanus subsp. clausenae' was the predominant Liberibacter detected in citrus showing mottling symptoms in Tororo district in Uganda and Kisumu and Kericho counties in Kenya, all sites at altitudes above 1,000 m. ' $\mathrm{Ca}$. L. africanus subsp. clausenae' was also found to infect citrus trees in the Morogoro region in Tanzania, but ' $C a$. L. africanus' was the predominant Liberibacter detected in samples from this area where altitudes were closer to $500 \mathrm{~m}$.

Trioza erytreae and Diaphorina citri samples from two collection sites. (Data not presented.) The identity of T. erytreae adults collected in Kericho County in Kenya and D. citri adults 
collected from the Morogoro region in Tanzania, both from citrus showing mottling symptoms, were determined morphologically. Three $T$. erytreae samples tested positive for ' $\mathrm{Ca}$. L. africanus subsp. clausenae' based on $16 \mathrm{~S}$ rDNA and $r p l J$ gene region sequences. This correlated to ' $\mathrm{Ca}$. L. africanus subsp. clausenae' detected in the citrus samples from which the adults were collected. Similarly, ' $\mathrm{Ca}$. L. africanus subsp. clausenae' was identified in two $D$. citri adults collected from a site in Tanzania where citrus samples tested positive for ' $C a$. L. africanus subsp. clausenae'.

\section{Discussion}

Samples collected from Uganda, Kenya, and Tanzania showing leaf mottling, typical for HLB, contained either ' $\mathrm{Ca}$. L. africanus' or ' $C a$. L. africanus subsp. clausenae'. The citrus trees sampled were either sweet orange or rough lemon, which was the only rootstock in use, but as most trees had been harvested at the time of the survey, it was not always obvious what the trees were. ' $\mathrm{Ca}$. L. africanus' was detected from several samples showing blotchy mottle symptoms at medium to high altitudes (1,090 $\mathrm{m}$ to 2,090 $\mathrm{m}$ in Uganda and Kenya and $525 \mathrm{~m}$ to $668 \mathrm{~m}$ in Tanzania). This finding is consistent with the known occurrence of this Liberibacter sp. in cooler, higher altitude regions. The presence of ' $\mathrm{Ca}$. L. asiaticus' in these regions was not confirmed by sequencing data from any of the samples tested, despite their testing positive for ' $\mathrm{Ca}$. L. asiaticus' with real-time PCR. These samples were all shown to contain ' $\mathrm{Ca}$. L. africanus subsp. clausenae' and were also from areas of medium to high altitudes, which are associated with cooler temperatures compared with lower lying regions. This would suggest that ' $C a$. L. africanus subsp. clausenae', like ' $\mathrm{Ca}$. L. africanus', is heat sensitive. Five samples, one from Uganda, one from Kenya, and three from Tanzania were shown

Table 2. Citrus survey samples, the respective collection locations and altitudes, and the Liberibacter spp. detected based on real-time PCR, sequence data, and 'Candidatus Liberibacter africanus'/'Ca. L. africanus subsp. clausenae' specific PCR assays. The target gene region of each assay is indicated in brackets.

\begin{tabular}{|c|c|c|c|c|c|c|c|c|}
\hline \multirow[b]{2}{*}{ Sample } & \multirow[b]{2}{*}{ Country } & \multirow[b]{2}{*}{ Region } & \multirow[b]{2}{*}{ Altitude (m) } & \multicolumn{2}{|c|}{$\begin{array}{c}\text { Real-time PCR } \\
\text { (Ct) (16S rDNA) }\end{array}$} & \multirow{2}{*}{$\begin{array}{l}\text { Sequence identity } \\
\quad(r p l J \text { and } o m p)\end{array}$} & \multicolumn{2}{|c|}{$\begin{array}{c}\text { Specific PCR } \\
(o m p)^{\mathrm{a}}\end{array}$} \\
\hline & & & & $\mathbf{L a f}^{\mathbf{b}}$ & $\overline{\operatorname{Las}^{c}}$ & & Laf & $\mathrm{LafCl}^{\mathrm{d}}$ \\
\hline \multicolumn{9}{|c|}{ First survey } \\
\hline 1 & Uganda & Budaka & 1,145 & 24.3 & - & Laf & + & - \\
\hline 2 & & & & 22.1 & - & Laf & + & - \\
\hline 3 & & & & 21.8 & - & Laf & + & - \\
\hline 4 & & & & - & 27.4 & $\mathrm{LafCl} / x^{\mathrm{e}}$ & + & + \\
\hline 5 & & Budaka & 1,154 & - & 25.9 & $\mathrm{LafCl}$ & - & + \\
\hline 6 & & & & 24.9 & - & Laf & + & - \\
\hline 7 & & & & 23.4 & - & Laf & + & - \\
\hline 8 & & Budaka & 1,134 & - & - & - & - & - \\
\hline 9 & & Tororo & 1,226 & - & - & - & - & - \\
\hline 10 & & Tororo & 1,134 & - & 23.3 & $\mathrm{LafCl}$ & - & + \\
\hline 11 & & & & - & 25.6 & $\mathrm{LafCl}$ & - & + \\
\hline 12 & & Tororo & 1,140 & - & 27.7 & $\mathrm{LafCl}$ & - & + \\
\hline 13 & & & & - & 27.3 & $\mathrm{LafCl}$ & - & + \\
\hline 14 & & Tororo & 1,098 & - & 26.8 & $\mathrm{LafCl}$ & - & + \\
\hline 15 & & & & - & 27.8 & $\mathrm{LafCl}$ & - & + \\
\hline 16 & & Tororo & 1,096 & - & 29.2 & $\mathrm{LafCl}$ & - & + \\
\hline 17 & & & & - & - & - & - & - \\
\hline 18 & & Tororo & 1,090 & - & 30.4 & $\mathrm{LafCl}$ & - & + \\
\hline 19 & Kenya & Kisumu & 1,147 & - & - & - & - & - \\
\hline 20 & & Kisumu & 1,146 & - & 27.9 & $\mathrm{LafCl}$ & - & + \\
\hline 21 & & Kisumu & 1,186 & - & 28.4 & $\mathrm{LafCl}$ & - & + \\
\hline 22 & & Kericho & 1,996 & - & 25.2 & $\mathrm{LafCl}$ & - & + \\
\hline 23 & & & & - & 26.9 & $\mathrm{LafCl}$ & - & + \\
\hline 24 & & Nakuru & 2,090 & - & - & Laf & + & + \\
\hline \multicolumn{9}{|c|}{ Second survey } \\
\hline 1 & Tanzania & Morogoro & 396 & - & - & - & - & - \\
\hline 2 & & Morogoro & 525 & 25.7 & - & Laf & + & - \\
\hline 3 & & & & 23.7 & - & Laf & + & - \\
\hline 4 & & & & 26.7 & 29.7 & Laf & + & + \\
\hline 5 & & & & 25.8 & - & Laf & + & - \\
\hline 6 & & Morogoro & 528 & - & 29.2 & $\mathrm{LafCl}$ & - & + \\
\hline 7 & & Morogoro & 574 & 30.3 & 29.8 & $\mathrm{LafCl}$ & + & + \\
\hline 8 & & & & 26.7 & - & Laf & + & - \\
\hline 9 & & Morogoro & 551 & 23.5 & - & Laf & + & - \\
\hline 10 & & Morogoro & 668 & 26.8 & 29.3 & Laf & + & + \\
\hline 11 & & & & - & 28.9 & $\mathrm{LafCl}$ & - & + \\
\hline 12 & & Chalinze & 222 & - & - & - & - & - \\
\hline 13 & & Zanzibar & 42 & - & - & - & - & - \\
\hline 14 & & Zanzibar & 48 & - & - & - & - & - \\
\hline 15 & & Kibiti & 111 & - & - & - & - & - \\
\hline 16 & & Mkuranga & 127 & - & - & - & - & - \\
\hline 17 & & Dar es Salaam & 19 & - & - & - & - & - \\
\hline
\end{tabular}


to be coinfected with both ' $C a$. L. africanus' and ' $C a$. L. africanus subsp. clausenae'. During a study aimed at characterizing the agent associated with HLB of citrus in Kenya, Magomere et al. (2009) found that the Liberibacter commonly associated with these trees formed a cluster highly similar to, but distinct from ' $\mathrm{Ca}$. L. africanus' based on phylogeny of L10/L12 rDNA sequences. As ' $\mathrm{Ca}$. L. africanus subsp. clausenae' was not known at that time, no correlation could be made between these samples and ' $\mathrm{Ca}$. L. africanus subsp. clausenae', but it

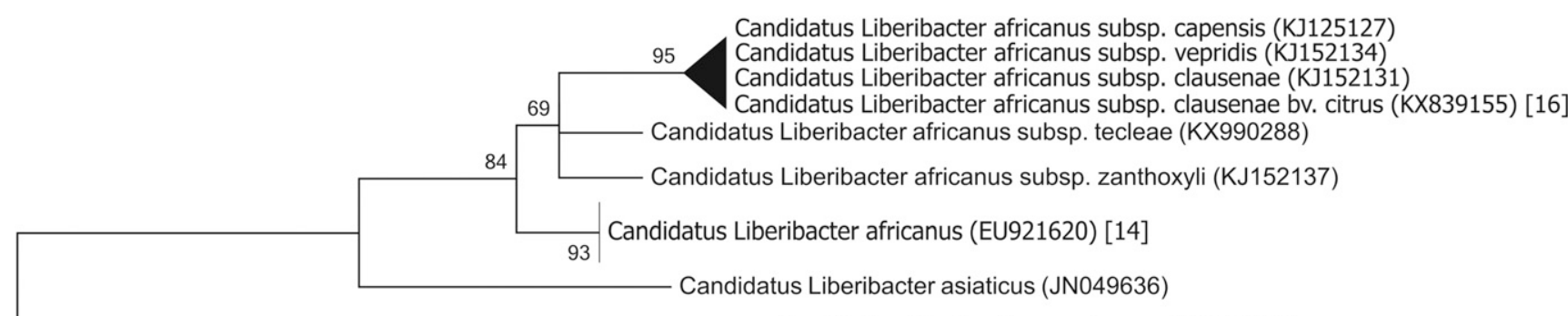

Candidatus Liberibacter americanus (AY742824)

0,0050

Fig. 1. Phylogeny of citrus-infecting members of the genus Liberibacter based on maximum-likelihood analysis of 16S rDNA sequences from symptomatic citrus samples from East Africa compared with various subspecies of 'Candidatus Liberibacter africanus'. Phylogeny was inferred using the Kimura-2-parameter model (Kimura 1980) with gamma corrections to account for among-site rate variation. Bootstrap values based on 1,000 replicates are shown at branch nodes. GenBank accessions for sequences used within this analysis are shown on the tree and the number of samples corresponding with homologous sequences are indicated in brackets. Bar, 0.0050 substitutions per nucleotide position.

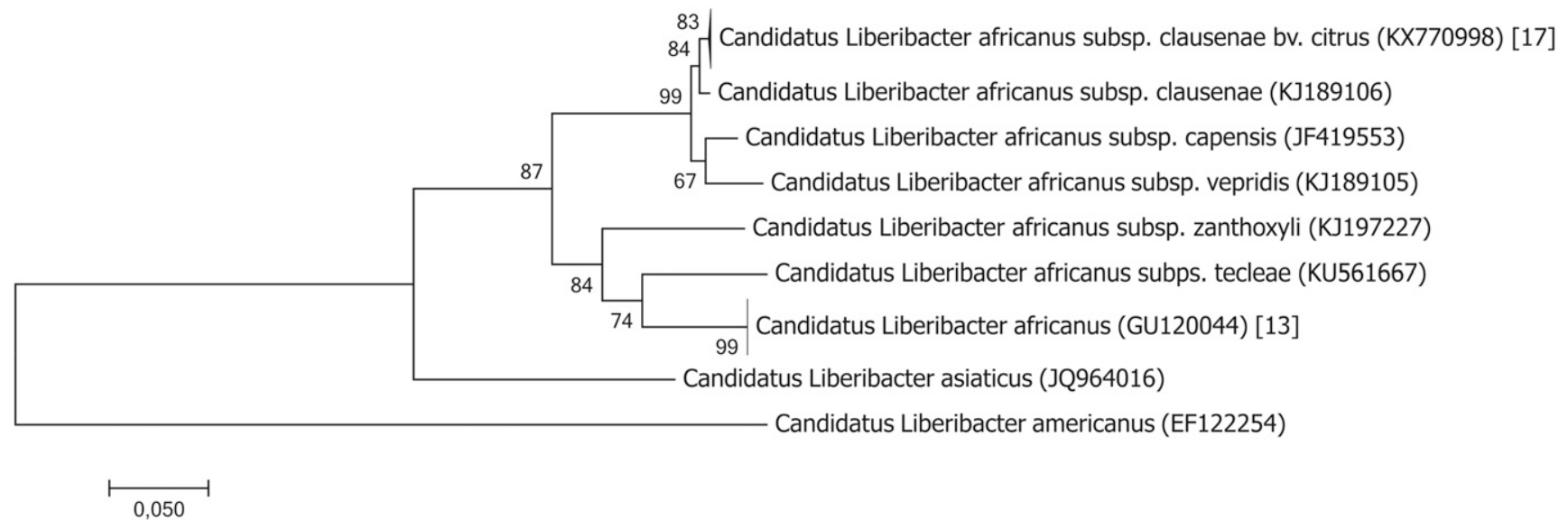

Fig. 2. Phylogeny of citrus-infecting members of the genus Liberibacter based on maximum-likelihood analysis of the rplJ gene sequences obtained from symptomatic citrus samples from East Africa compared with various subspecies of 'Candidatus Liberibacter africanus'. Phylogeny was inferred using the Hasegawa-Kishino-Yano model (Hasegawa et al. 1985) with gamma corrections to account for among-site rate variation. Bootstrap values based on 1,000 replicates are shown at branch nodes. GenBank accessions for sequences used within this analysis are shown on the tree and the number of samples corresponding with homologous sequences are indicated in brackets. Bar, 0.050 substitutions per nucleotide position.

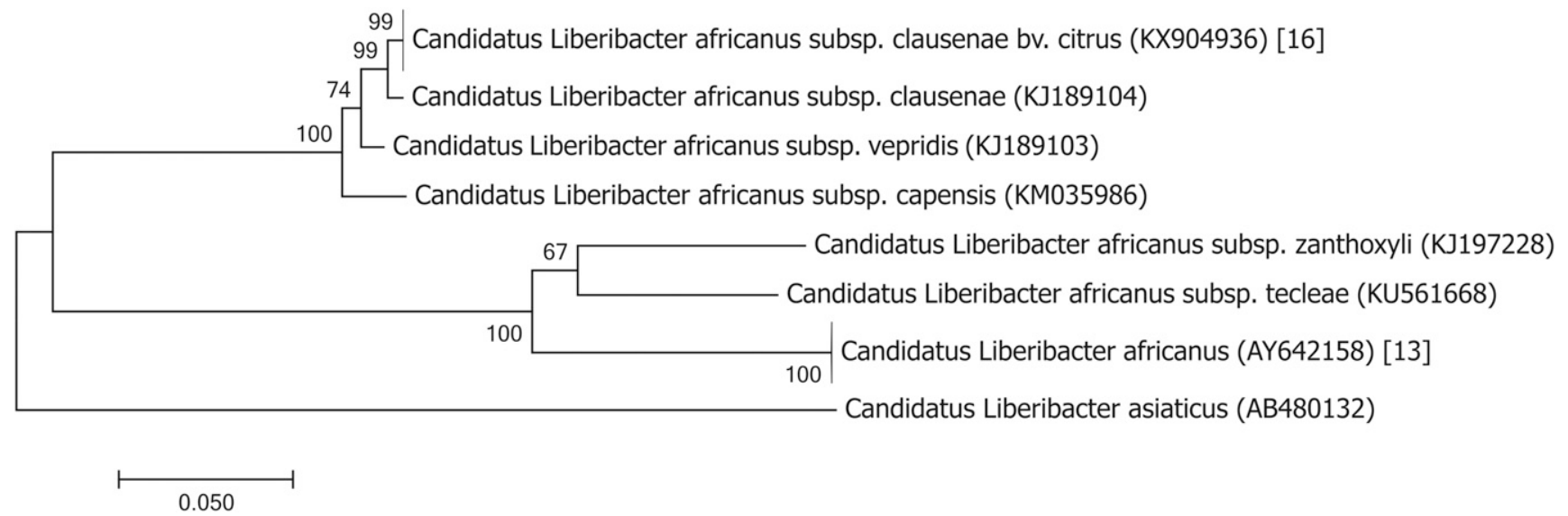

Fig. 3. Phylogeny of citrus-infecting members of the genus Liberibacter based on maximum-likelihood analysis of the omp gene sequences obtained from symptomatic citrus samples from East Africa compared with various subspecies of 'Candidatus Liberibacter africanus'. Phylogeny was inferred using the Hasegawa-Kishino-Yano model (Hasegawa et al. 1985) with gamma corrections to account for among-site rate variation. Bootstrap values based on 1,000 replicates are shown at branch nodes. GenBank accessions for sequences used within this analysis are shown on the tree and the number of samples corresponding with homologous sequences are indicated in brackets. Bar, 0.050 substitutions per nucleotide position. 
is likely that the agent identified by Magomere et al. (2009) was ' $\mathrm{Ca}$. L. africanus subsp. clausenae'. The predominant Liberibacter identified with HLB-affected trees in Kenya during this study was also ' $\mathrm{Ca}$. L. africanus subsp. clausenae'. No mottling symptoms were observed at warmer, lower altitude coastal regions in Tanzania or eastern Kenya, which would be expected if ' $\mathrm{Ca}$. L. asiaticus' was present.

The unconfirmed detection of ' $\mathrm{Ca}$. L. asiaticus' from Tanzania (Shimwela et al. 2016) may also be explained by the nontarget amplification observed for ' $\mathrm{Ca}$. L. africanus subsp. clausenae' with the real-time PCR assay of Li et al. (2006) for ' $\mathrm{Ca}$. L. asiaticus' detection. Our results therefore indicated that ' $\mathrm{Ca}$. L. africanus subsp. clausenae' is also amplified with ' $\mathrm{Ca}$. L. asiaticus' primers, as all samples positive for ' $C a$. L. asiaticus' following real-time PCR were demonstrated to be infected with ' $\mathrm{C} a$. L. africanus subsp. clausenae' when sequenced. Nontarget amplification was also demonstrated for the known subspecies of ' $\mathrm{Ca}$. L. africanus' identified from South Africa. The ' $\mathrm{Ca}$. L. africanus' subspecies used to test the specificity of the real-time assays were obtained from indigenous rutaceous trees collected from various localities in South Africa. As ' $\mathrm{Ca}$. L. asiaticus' is not present in South Africa (Pietersen et al. 2010) and has not been identified from rutaceous hosts other than citrus,
Murraya, and Severinia (Deng et al. 2008; Lopes et al. 2010; Walter et al. 2012), any positive detection can more likely be attributed to nontarget amplification and not the presence of ' $\mathrm{Ca}$. L. asiaticus'. Amplification was also obtained for the known subspecies of ' $\mathrm{Ca}$. $\mathrm{L}$. africanus' in rutaceous hosts with the ' $\mathrm{Ca}$. L. africanus' real-time primers, but not from citrus samples containing ' $\mathrm{Ca}$. L. africanus subsp. clausenae'. This difference could possibly be due to a lower titer of ' $C a$. L. africanus subsp. clausenae' in the citrus host compared with C. anisata, but this requires further verification. The results from the real-time specificity assay also suggest that the ' $\mathrm{Ca}$. L. asiaticus' primers detect the ' $\mathrm{Ca}$. L. africanus' subspecies more efficiently compared with the ' $\mathrm{Ca}$. L. africanus' primers, given the consistently lower $\mathrm{Ct}$ values for the same samples. The nontarget amplification of the ' $\mathrm{Ca}$. L. africanus'-subspecies demonstrated for both the ' $\mathrm{Ca}$. L. africanus' and 'Ca. L. asiaticus' assays of Li et al. (2006) is likely due to the $16 \mathrm{~S}$ rDNA target region of these assays. The highly conserved nature of this gene region among citrus-infecting Liberibacter species has been noted previously (Ghosh et al. 2013; Roberts et al. 2015). We propose that detection assays target less conserved genomic regions to prevent false positive detection of ' $\mathrm{Ca}$. L. asiaticus'. From an African perspective, it is also important that tests be able

Table 3. Collection localities of samples containing various 'Candidatus Liberibacter africanus' subspecies and results obtained with 'Ca. L. africanus' and 'Ca. L. asiaticus' real-time PCR assays

\begin{tabular}{|c|c|c|c|c|c|}
\hline \multirow[b]{2}{*}{ Liberibacter } & \multirow[b]{2}{*}{ Host } & \multirow[b]{2}{*}{ Country / Province } & \multirow[b]{2}{*}{ Locality } & \multicolumn{2}{|c|}{ Real-time PCR (Ct value $\left.{ }^{a}\right)$} \\
\hline & & & & Laf $^{\mathbf{b}}$ & $\operatorname{Las}^{\mathbf{c}}$ \\
\hline & & South Africa & & & \\
\hline LafC $^{d}$ & Calodendrum capense & Eastern Cape & Kirkwood & 17.5 & 14.9 \\
\hline LafC & Calodendrum capense & Eastern Cape & Kirkwood & 17.3 & 14.4 \\
\hline LafC & Calodendrum capense & Mpumalanga & Nelspruit & 19.7 & 15.8 \\
\hline $\mathrm{LafCl}^{\mathrm{e}}$ & Clausena anisata & Western Cape & Jongensfontein & 22.3 & 18.4 \\
\hline $\mathrm{LafCl}$ & Clausena anisata & KwaZulu-Natal & Pietermaritzburg & 21.7 & 18.8 \\
\hline $\mathrm{LafCl}$ & Clausena anisata & Mpumalanga & Barberton & 18.5 & 14.9 \\
\hline $\mathrm{LafV}^{\mathrm{f}}$ & Vepris lanceolata & Eastern Cape & Grahamstown & 25.0 & 23.3 \\
\hline LafV & Vepris lanceolata & Eastern Cape & Grahamstown & 27.7 & 26.6 \\
\hline LafV & Vepris lanceolata & Western Cape & Knysna & 22.0 & 17.8 \\
\hline $\mathrm{LafZ}^{\mathrm{g}}$ & Zanthoxylum dayvii & KwaZulu-Natal & Kokstad & - & - \\
\hline LafZ & Zanthoxylum capense & Western Cape & Knysna & 27.8 & 24.8 \\
\hline LafZ & Zanthoxylum capense & Western Cape & Knysna & 26.1 & 24.2 \\
\hline Laf Control & Citrus sinensis & Gauteng & Pretoria & 23.2 & - \\
\hline Las Control & Citrus sinensis & Ethiopia & & - & 19.6 \\
\hline
\end{tabular}

a The average standard deviation for samples in the 'Ca. L. africanus' assay was 0.28 and 0.22 for samples in the ' $\mathrm{Ca}$. L. asiaticus' assay.

b 'Ca. L. africanus'.

c 'Ca. L. asiaticus'.

d ' $C a$. L. africanus subsp. capensis'.

e 'Ca. L. africanus subsp. clausenae'.

f 'Ca. L. africanus subsp. vepridis'.

g 'Ca. L. africanus subsp. zanthoxyli’.

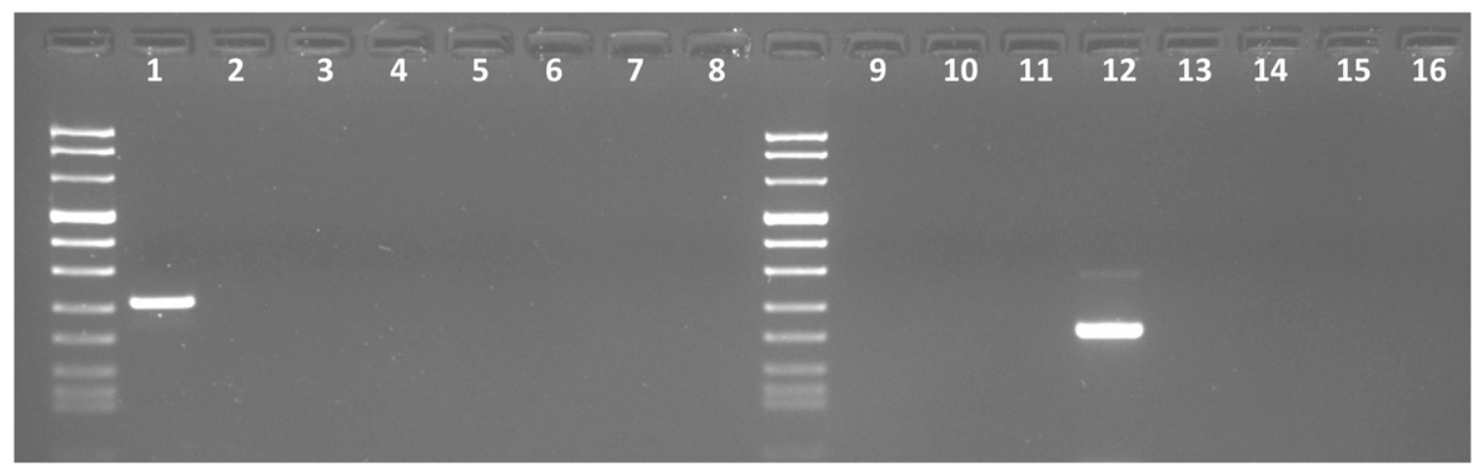

Fig. 4. Electropherogram of 'Candidatus Liberibacter africanus' and 'Ca. L. africanus subsp. clausenae' specific end point PCR reactions using 'Ca. L. africanus' and 'Ca. L. africanus subsp. clausenae' specific primers. Lanes 1-8 show amplification of various Liberibacters using 'Ca. L. africanus' specific primers and lanes 9-16 show amplification using 'Ca. L. africanus subsp. clausenae' specific primers. The Liberibacter represented per lane is as follows: 1 and 9 ' Ca. L. africanus', 2 and 10 'Ca. L. asiaticus', 3 and 11 'Ca. L. africanus subsp. capensis', 4 and 12 'Ca. L. africanus subsp. clausenae', 5 and 13 'Ca. L. africanus subsp. vepridis', 6 and 14 'Ca. L. africanus subsp. zanthoxyli'. Lanes 7 and 15 contain a healthy citrus control and lanes 8 and 16 are no template controls. Unlabeled lanes contain DNA molecular Marker VIII (Roche). 
to differentiate not only between ' $\mathrm{C}$. L. africanus', ' $\mathrm{Ca}$. L. asiaticus', and ' $\mathrm{Ca}$. L. americanus', but the various subspecies of ' $\mathrm{Ca}$. L. africanus'. In this study, detection assays based on respective ' $\mathrm{Ca}$. L. africanus' and ' $\mathrm{Ca}$. L. africanus subsp. clausenae' omp sequences were successfully developed and were used to confirm that coinfection of citrus with ' $\mathrm{Ca}$. L. africanus' and ' $\mathrm{Ca}$. L. africanus subsp. clausenae' had occurred. This study shows that ' $\mathrm{Ca}$. L. asiaticus' is not present in Tanzania and is not the causative agent for HLB in the country as earlier reported by Shimwela et al. (2016).

Detection of ' $C a$. L. asiaticus' in Uganda by Kalyebi et al. (2015) was based on Acceler8 AmplifyRP isothermal amplification platform (Agdia), but it is not clear which genomic regions were targeted for amplification with this test. While we cannot therefore infer potential nonspecific detection, the present study included samples from the same regions in Uganda (Budaka and Tororo) where ' $\mathrm{Ca}$. L. asiaticus' had been reported. Only ' $\mathrm{Ca}$. L. africanus' and ' $\mathrm{Ca}$. L. africanus subsp. clausenae' were detected in Budaka and ' $C a$. L. africanus subsp. clausenae' in Tororo, but no ' $C a$. L. asiaticus' was detected in either region. We therefore strongly suspect that the report of ' $\mathrm{Ca}$. L. asiaticus' in Uganda (Kalyebi et al. 2015) could be a similar misidentification as demonstrated with the $\mathrm{Li}$ et al. (2006) assay. We additionally visited regions in Kenya adjacent to those where the putative positive reports were made, including the counties of Kisumu, Kericho, and Nakuru, and again, ' $\mathrm{Ca}$. L. africanus subsp. clausenae' and not ' $\mathrm{Ca}$. L. asiaticus' was detected from these sites.

The ' $C a$. L. africanus subsp. clausenae' sequences obtained from citrus were not identical to ' $\mathrm{Ca}$. L. africanus subsp. clausenae' $r p l J$ and $o m p$ sequences obtained from $C$. anisata. SNPs observed for ' $C a$. L. africanus subsp. clausenae' sequences from citrus occurred at the same nucleotide positions for all citrus samples testing positive for ' $C a$. L. africanus subsp. clausenae', supporting designation of ' $\mathrm{Ca}$. L. africanus subsp. clausenae' from citrus as a separate biovar to ' $\mathrm{Ca}$. L. africanus subsp. clausenae' from Clausena.

C. anisata is indigenous to Africa and ' $\mathrm{Ca}$. L. africanus subsp. clausenae' was initially identified from seemingly healthy trees in South Africa, indicating a long-standing association between ' $\mathrm{C} a$. L. africanus subsp. clausenae' and this host species (Roberts et al. 2015). However, citrus trees found to be positive for ' $\mathrm{Ca}$. L. africanus subsp. clausenae' showed typical mottling symptoms associated with HLB disease, suggesting that this association occurred more recently. During surveys, no $C$. anisata was observed near the citrus trees sampled, despite being known to occur in the East African countries surveyed (Duncan and Chapman 2003; Innocent and Hassanali 2015; Kigomo et al. 1990). The absence of $C$. anisata near cultivated citrus orchards further suggests a possible selective adaptation of ' $\mathrm{Ca}$. L. africanus subsp. clausenae' to its new citrus host by changes at the molecular level, resulting in the biovar found in this study.

' $C a$. L. africanus subsp. clausenae' $r p l J$ and $16 \mathrm{~S}$ rDNA sequences were additionally confirmed from DNA extracted from both $D$. citri and $T$. erytreae in the current study. These results imply that these pests could be potential vectors for ' $\mathrm{Ca}$. L. africanus subsp. clausenae'; however, transmission studies will be required to confirm these as vectors.

In conclusion, ' $\mathrm{Ca}$. L. asiaticus' was not detected from any of the citrus trees or psyllids sampled during the current survey, indicating that the initial tests by Kalyebi et al. (2015) and Shimwela et al. (2016) are likely due to a nontarget amplifications with ' $\mathrm{Ca}$. L. africanus subsp. clausenae', as demonstrated with the Li et al. (2006) assay. It is vital that a new test for ' $\mathrm{Ca}$. L. asiaticus' be developed that does not amplify from the various nontarget Liberibacters present in Africa. This is also the first conclusive report of a biovar of ' $\mathrm{Ca}$. $\mathrm{L}$. africanus subsp. clausenae' being associated with HLB symptoms in Africa, giving insight into the involvement of ' $\mathrm{Ca}$. L. africanus' subspecies in citriculture in Africa.

\section{Acknowledgments}

We dedicate this publication to our co-author Dr. Hennie le Roux, who initiated this study, but who sadly passed away before the manuscript could be completed.
We wish to acknowledge his driving passion for the citrus industry that he served. We would also like to thank Citrus Research International and the icipe GIZ funded Strengthening Citrus IPM project for funding and the National Research Foundation for providing the funding for sequencing.

\section{Literature Cited}

Bastianel, C., Garnier-Semancik, M., Renaudin, J., Bové, J. M., and Eveillard, S. 2005. Diversity of 'Candidatus Liberibacter asiaticus' based on the omp gene sequence. Appl. Environ. Microbiol. 71:6473-6478.

Bové, J. M. 2014. Heat tolerant Asian HLB meets heat-sensitive African HLB in the Arabian Peninsula! Why? J. Citrus Pathol. 1:1-78.

Buitendag, C. H., and von Broembsen, L. A. 1993. Living with Citrus Greening in South Africa. Pages 269-273 in: Twelfth IOCV Conference. P. Moreno, J. V. da Graca, and L. W. Timmer, eds. IOCV, New Delhi, India.

Capoor, S. P., Rao, D. G., and Viswanath, S. M. 1967. Diaphorina citri Kuwayama, a vector of the greening disease of citrus in India. Indian J. Agric. Sci. 37:572-575.

Catling, H. D. 1969. The bionomics of the South African citrus psylla, Trioza erytreae (Del Guercio) (Homoptera: Psyllidae) 3. The influence of extremes of weather on survival. J. Entomol. Soc. South. Afr. 32:273-290.

Coletta-Filho, H. D., Targon, M. L. P. N., Takita, M. A., de Negri, J. D., Pompeu, J., Jr., Machado, M. A., do Amaral, A. M., and Muller, G. W. 2004. First report of the causal agent of Huanglongbing ('Candidatus Liberibacter asiaticus) in Brazil. Plant Dis. 88:1382.

da Graça, J. V. 1991. Citrus greening disease. Annu. Rev. Phytopathol. 29: $109-136$

da Graça, J. V., Kunta, M., Sétamou, M., Rascoe, J., Li, W., Nakhla, M. K., Salas, B., and Bartels, D. W. 2015. Huanglongbing in Texas: Report on the first detections in commercial citrus. J. Citrus Pathol. 2:1.

Davis, R. I., Jacobson, S. J., Rahamma, S., and Gunua, T. G. 2000. Surveillance for citrus Huanglongbing (greening) disease in New Guinea and North Queensland. Australas. Plant Pathol. 29:226

Deng, X., Lou, Z., Feng, Z., Li, H., Chen, J., and Civerolo, E. L. 2008. First Report of 'Candidatus Liberibacter asiaticus' from Atalantia buxifolia in Guangdong, China. Plant Dis. 92:314.

Doyle, J. J., and Doyle, J. L. 1990. Isolation of plant DNA from fresh tissue. Focus 12:13-15

Duncan, R. S., and Chapman, C. A. 2003. Tree-shrub interactions during early secondary forest succession in Uganda. Restor. Ecol. 11:198-207.

Garnier, M., and Bové, J. M. 1996. Distribution of the Huanglongbing (greening) Liberobacter species in fifteen African and Asian countries. Pages 388-391 in: Proceedings of the Thirteenth Conference of the International Organization of Citrus Virologists. J. V da Graça, R. F. Lee, and R. K. Yokomi, eds. IOCV, Fuzhou, China.

Garnier, M., Jagoueix, S., Toorawa, P., Grisoni, M., Mallesard, R., Dookun, A., Saumtally, S., Autrey, J. C., and Bové, J. M. 1996. Both Huanglongbing (greening) Liberobacter species are present in Mauritius and Réunion. Pages 271-275 in: Proceedings of the Thirteenth Conference of the International Organization of Citrus Virologists. J. V da Graça, R. F. Lee, and R. K. Yokomi, eds. IOCV, Fuzhou, China.

Garnier, M., Jagoueix-Eveillard, S., Cronje, P. R., Le Roux, H. F., and Bové, J. M. 2000. Genomic characterization of a Liberibacter present in an ornamental rutaceous tree, Calodendrum capense, in the Western Cape province of South Africa. Proposal of 'Candidatus Liberibacter africanus subsp. capensis'. Int. J. Syst. Evol. Microbiol. 50:2119-2125.

Ghosh, D., Bhose, S., Mukherjee, K., and Baranwal, V. K. 2013. Sequence and evolutionary analysis of Ribosomal DNA from huanglongbing (HLB) isolates in Western India. Phytoparasitica 41:295-305.

Gottwald, T. R., da Graça, J. V., and Bassanezi, R. B. 2007. Citrus Huanglongbing: The pathogen and its impact. Online. Plant Health Prog. doi:10.1094/PHP2007-0906-01-RV

Gravena, S., Beretta, M. J. G., Paiva, P. E. B., Gallã, R., and Yamamoto, P. T 1996. Seasonal abundance and natural enemies of Diaphorina citri (Hemiptera: Psyllidae) in citrus orchards of São Paulo State, Brazil. Page 414 in: Proceedings of the Thirteenth Conference of the International Organization of Citrus Virologists. J. V da Graça, R. F. Lee, and R. K. Yokomi, eds. IOCV, Fuzhou, China.

Halbert, S. E. 2005. The discovery of Huanglongbing in Florida. In: Proceedings of the Second International Citrus Canker and Huanglongbing Research Workshop, abstract H-3. Florida Citrus Mutual, Orlando, FL.

Hall, T. A. 1999. BioEdit: a user-friendly biological sequence alignment editor and analysis program for windows 95/98/NT. Nucleic Acids Symp. Ser. 41:95-98

Hasegawa, M., Kishino, H., and Yano, T. 1985. Dating the human-ape split by a molecular clock of mitochondrial DNA. J. Mol. Evol. 22:160-174.

Hocquellet, A., Toorawa, P., Bové, J. M., and Garnier, M. 1999. Detection and identification of the two Candidatus Liberobacter species associated with citrus Huanglongbing by PCR amplification of ribosomal protein genes of the $\beta$ operon. Mol. Cell. Probes 13:373-379.

Innocent, E., and Hassanali, A. 2015. Constituents of essential oils from three plant species used in traditional medicine and insect control in Tanzania. J. Herbs Spices Med. Plants 21:219-229. 
Jagoueix, S., Bové, J. M., and Garnier, M. 1994. The phloem-limited bacterium of greening disease of citrus is a member of the $\alpha$-subdivision of the Proteobacteria. Int. J. Syst. Bacteriol. 44:379-386.

Jagoueix, S., Bové, J. M., and Garnier, M. 1996. PCR detection of the two 'Candidatus' Liberobacter species associated with greening disease of citrus. Mol. Cell. Probes 10:43-50.

Kalyebi, A., Aisu, G., Ramathani, J., Ogwang, J., McOwen, N., and Russell, P. 2015. Detection and identification of etiological agents (Liberibacter spp.) associated with citrus greening disease in Uganda. Uganda J. Agric. Sci. 16: 43-54.

Kapur, S. P., Kapoor, S. K., Cheema, S. S. and Dhillon, R. S. 1978. Effect of greening disease on tree and fruit characters of Kinnow mandarin. Punjab Hortic. J. 18:76-79.

Katoh, K., Misawa, K., Kuma, K., and Miyata, T. 2002. MAFFT: a novel method for rapid multiple sequence alignment based on fast fourier transform. Nucleic Acids Res. 30:3059-3066.

Kigomo, B. N., Savill, P. S., and Woodell, S. R. 1990. Forest composition and its regeneration dynamics; a case study of semi-deciduous tropical forests in Kenya. Afr. J. Ecol. 28:174-188.

Kimura, M. 1980. A simple method for estimating evolutionary rates of base substitutions through comparative nucleotide sequences. J. Mol. Evol. 16: 111-120.

Knapp, J. L., Halbert, S., Lee, R., Hoy, M., Clark, R., and Kesinger, M. 1998. The Asian citrus psyllid and citrus greening disease. Citrus Ind. 79:28-29.

Kumar, S., Stecher, G., and Tamura, K. 2016. MEGA7: Molecular evolutionary genetic analysis version 7.0 for Bigger Datasets. Mol. Biol. Evol. 33: 1870-1874.

Lallemand, J., Fos, A., and Bové, J. M. 1986. Transmission de la bacteria associé à la forme africaine de la maladie du "greening" par le psylle asiatique Diaphorina citri Kuwayama. Fruits 41:341-343.

Li, W., Hartung, J. S., and Levy, L. 2006. Quantitative real-time PCR for the detection amd identification of Candidatus Liberibacter species associated with citrus Huanglongbing. J. Microbiol. Methods 66:104-115.

Lopes, S. A., Frare, G. F., Camargo, L. E. A., Wulff, N. A., Teixeira, D. C., Bassanezi, R. B., Beattie, G. A. C., and Ayres, A. J. 2010. Liberibacters associated with orange jasmine in Brazil: incidence in urban areas and relatedness to citrus Liberibacters. Plant Pathol. 59:1044-1053.

Magomere, T. O., Obukosia, S. D., Mutitu, E., Ngichabe, C., Olubayo, F., and Shibairo, S. 2009. Molecular characterization of 'Candidatus Liberibacter' species/strains causing huanglongbing disease of citrus in Kenya. Electron. J. Biotechnol. 12. doi:10.2225/vol12-issue2-fulltext-2.

Massonie, G., Garnier, M., and Bové, J. M. 1976. Transmission of Indian citrus decline by Trioza erytreae (del Guercio), the vector of South African greening. Pages 18-20 in: Proceedings of the Seventh Conference of the International Organization of Citrus Virologists. E. C. Calavan, ed. IOCV, Athens, Greece.

McClean, A. P. D., and Oberholzer, P. C. J. 1965. Citrus psylla, a vector of the greening disease of sweet orange. S. Afr. J. Agric. Sci. 8:297-298.
Oberholzer, P. C. J., von Staden, D. F. A., and Basson, W. J. 1963. Greening disease of sweet orange in South Africa. Pages 213-219 in: Proceedings of the Third Conference of the International Organization of Citrus Virologists. W. C. Price, ed. IOCV, São Paulo, Brazil.

Pietersen, G., Arrebola, E., Breytenbach, J. H. J., Korsten, L., le Roux, H. F., la Grange, H., Lopes, S. A., Meyer, J. B., Pretorius, M. C., Schwerdtfeger, M., van Vuuren, S. P., and Yamamoto, P. 2010. A survey of 'Candidatus Liberibacter' species in South Africa confirms the presence of only ' $\mathrm{Ca}$. L. africanus' in commercial citrus. Plant Dis. 94:244-249.

Pretorius, M. C., and van Vuuren, S. P. 2006. Managing Huanglongbing (Citrus greening disease) in the Western Cape. S. Afr. Fruit J. 5:59-62.

Roberts, R., and Pietersen, G. 2016. A novel subspecies of 'Candidatus Liberibacter africanus' found on native Teclea gerrardii (Family: Rutaceae) from South Africa. Antonie van Leeuwenhoek 110:437-444.

Roberts, R., Steenkamp, E. T., and Pietersen, G. 2015. Three novel lineages of 'Candidatus Liberibacter africanus' associated with native rutaceous hosts of Trioza erytreae in South Africa. Int. J. Syst. Evol. Microbiol. 65:723-731.

Saponari, M., De Bac, G., Breithaupt, J., Loconsole, G., Yokomi, R. K., and Catalano, L. 2010. First report of 'Candidatus Liberibacter asiaticus' associated with Huanglongbing in sweet orange in Ethiopia. Plant Dis. 94:482.

Schwarz, R. E. 1967. Results of a greening survey on sweet orange in the major citrusgrowing areas of the republic of South Africa. S. Afr. J. Agric. Sci. 10:471-476.

Schwarz, R. E., and Green, G. C. 1972. Heat Requirements for Symptom Suppression and Inactivation of the Greening Pathogen. Pages 44-51 in: Fifth IOCV Conference. W. C. Price, ed. IOCV, Tokyo, Japan.

Shimwela, M. M., Narouei-Khandan, H. A., Halbert, S. E., Keremane, M. L. Minsavage, G. V., Timilsina, S., Massawe, D. P., Jones, J. B., and van Bruggen, A. H. C. 2016. First occurrence of Diaphorina citri in East Africa, characterization of the $C a$. Liberibacter species causing Huanglongbing (HLB) in Tanzania, and potential further spread of D. citri and HLB in Africa and Europe. Eur. J. Plant Pathol. 146:349-368.

Teixeira, D. C., Ayres, J., Kitajima, E. W., Danet, L., Jagoueix-Eveillard, S., Saillard, C., and Bové, J. M. 2005. First report of a huanglongbing-like disease of citrus in São Paulo state, Brazil and association of a new liberibacter species, 'Candidatus Liberibacter americanus', with the disease. Plant Dis. 89:107.

Walter, A. J., Hall, D. G., and Duan, Y. P. 2012. Low incidence of 'Canditatus Liberibacter asiaticus' in Murraya paniculata and associated Diaphorina citri. Plant Dis. 96:827-832.

Weinert, M. P., Jacobson, S. C., Grimshaw, J. F., Bellis, G. A., Stephens, P. M., Gunua, T. G., Kame, M. F., and Davis, R. I. 2004. Detection of Huanglongbing (citrus greening disease) in Timor-Leste (East Timor) and Papua New Guinea. Australas. Plant Pathol. 33:135-136.

Zhang, M., Duan, Y., Zhou, L., Turecheck, W. W., Stover, E., and Powell, C. A 2010. Screening molecules for control of citrus Huanglongbing using an optimized regeneration system for 'Candidatus Liberibacter asiaticus'infected periwinkle (Catharanthus roseus) cuttings. Phytopathology 100: 239-245. 